九州大学学術情報リポジトリ

Kyushu University Institutional Repository

\title{
TWO-MODE TWO-FACTOR ANALYSIS
}

Asano, Choichiro

Research Institute of Fundamental Information Science, Kyushu University

https://doi.org/10.5109/13093

出版情報: 統計数理研究. 16 (3/4)，pp. 37-46，1975-03. Research Association of Statistical Sciences

バージョン：

権利関係 : 


\title{
TWO-MODE TWO-FACTOR ANALYSIS*
}

By

\author{
Chooichiro Asano**
}

(Received September 24, 1974)

\section{Summary.}

A factor-analytical method is proposed to evaluate two-way cross-classified observations. The suggested principle is based on a confirmatory situation of two-mode factor analysis in designed experimentation of physical sciences. The model has twomode interactive factors, which are defined by two two-factor loading matrices. The analysis and procedure are rather simple and a complete FORTRAN program is given in this paper. The advantage of the proposed method is immediately to make clear the information structure involved in observations, by means of two-mode experimental factor evaluation.

\section{Introduction.}

Evaluation of individual subjects may be designed by using a rating sheet, in which a $p$-dimensional set of traits is well-selected in view of the objective measurements of implied attempt. Then the ratings are obtained by such a manner that each subject in a given group is measured on each trait described in a sheet.

The usual methods of factor-analytical evaluation may extract several factors involved in a group of subjects or a set of traits, as in one-way allocation. That is to say, the analytical situations have been limited only in one-dimensional consideration such as $O_{-}, P_{-}, Q_{-}, R-, S_{-}$or $T$-technique in the sense of R. B. Cattell.

On the other hand, Levin and Tucker have attempted to extend such one-way models to two- or more-way factor-analytical models and procedures, since the factors are frequently interacted from both sides of subjects and traits. In this situation, a body of observations has a two-way cross-classification with some levels of subjects and some kinds of traits. The approach in this situation is called two-mode factor analysis in terminology. However, it may be possible to say that their methods are based on two principal component analyses applied from each side of two-way. Therefore, the methods are awfully explorative, since they still stand upon an unspecified model in view of the number of factors and the structures of loading matrices in both

* This study was supported by the Special Research Project No. 920213 of the Ministry of Education, "Advanced Information Processing of Large Scale Data over a Broad Area".

** Presently at Research Institute of Fundamental Information Science, Kyushu University, Fukuoka. This paper was written while the author was a research member of $\mathrm{C}-2$ : "The structure of data base and theory of information retrieval", (1974). 
sides of two-way cross-classification.

On several occasions, such observations in physical sciences may be obtained on the basis of an experimental design. We now assume such a design as a two-way layout with different numbers of levels. This implies that the meanning or interpretation of latent factors of both ways may be made confirmatorily clear under the suggested knowledge in science, and that in such designs of experiments a factoranalytical method is quantitatively needed in view of the scientific confirmation. The present paper starts straightforward from such situations of two-mode factor analysis, and gives a model and the analytical procedure. The factor-analytical method proposed here is of the most comfirmatory investigation, and in terminology may be call twomode two-factor analysis, where two-factor analysis is used in the sense of Spearman and Holzinger.

\section{The model.}

Let $X$ be a $p \times n$ data matrix, where the element $x_{i j}$ is an observation for the $i$-th trait and the $j$-th subject, $i=1,2, \cdots, p ; j=1,2, \cdots, n$. These $p$ traits and $n$ subjects are assumed to be designed in a cross-classification of two-way layout with $q$ categories of traits and $m$ categories of subjects. These categories are physically based on scientists' insight as to what aim they had made to plan the experiment, and this presents an essential situation of two-mode factor-analytic methods. We now denote such categories as $\mathfrak{P}_{1}, \mathfrak{P}_{2}, \cdots, \mathfrak{P}_{q}$, and $\mathfrak{R}_{1}, \mathfrak{N}_{2}, \cdots, \mathfrak{N}_{m}$, respectively, and further denote the numbers of items belonging to these categories as $p_{1}, p_{2}, \cdots, p_{q}$, and $n_{1}, n_{2}, \cdots, n_{m}$, where $p=\sum_{i=1}^{q} p_{i}$ and $n=\sum_{i=1}^{m} n_{i}$. Thus the present model of the data matrix is given by

$$
X=A C B+E,
$$

where $A$ and $B$ are real coefficient matrices $p \times q$ and $m \times n$ of two loading matrices for traits and subjects, $C$ is a core matrix which corresponds to a score matrix of the interactive two-way factors, and $E$ is an error matrix. As stated above, the elements of coefficient matrices, $A$ and $B$, in the present model are so arranged as to be direct products of $q$ column subvectors and of $m$ row subvectors with non-zero elements, respectively. That is to say, they are shown in the following way.
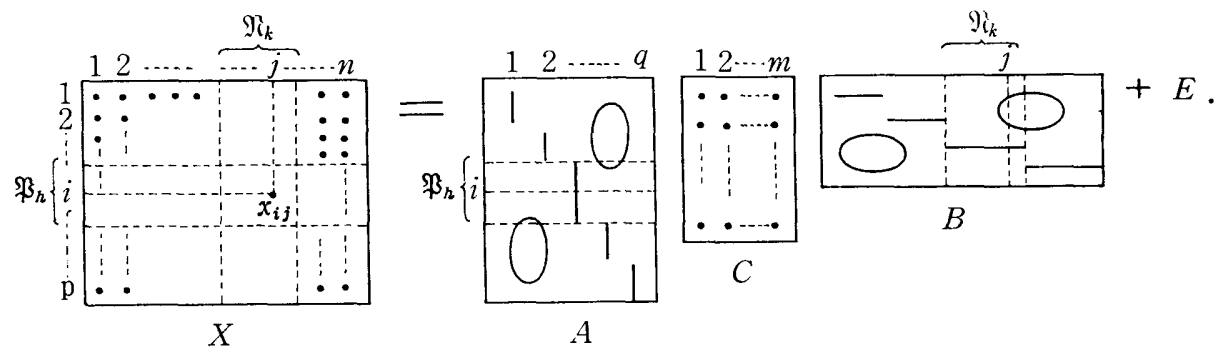

Thus the problem is to estimate the values of $A, B, C$ and $E$ under the condition of structure, staring from only a given data matrix like the usual methods of factor analyses, where we assume the following standardization for $A$ and $B$, without any 
loss of generality,

$$
A^{\prime} A=I_{q}, \quad B B^{\prime}=I_{m}
$$

\section{The analysis and procedure.}

Another convenient expression of the general model (1) of two-mode factor analysis is given element-wise by

$$
x_{i j}=\sum_{h=1}^{q} \sum_{k=1}^{m} a_{i n} c_{h k} b_{k j}+e_{i j}, \quad \begin{array}{ll}
i & =1,2, \cdots, p \\
& =1,2, \cdots, n
\end{array}
$$

where $e_{i j}$ is mutually independent for $i$ and $j$, and $E\left(e_{i j}\right)=0$.

In the present situation of the structure (2), the $x_{i j}$ is immediately shown as follows,

$$
x_{i j}=a_{i h} c_{h k} b_{k j}+e_{i j} \quad \text { for } \quad i \in \mathfrak{P}_{h}, j \in \mathfrak{\Re}_{k} .
$$

We shall now investigate asymptotically some parametric relationship for (5). Let $\phi_{i i^{\prime}}$ and $r_{i i}$, be a parameter and the sample observation of cross-product for the $i$-th and the $i^{\prime}$-th traits within a trait group in a category. Then we can express as follows,

$$
\begin{aligned}
\phi_{i i^{\prime}} & =E\left(r_{i i^{\prime}}\right)=E\left\{\sum_{j=1}^{n} x_{i j} x_{i^{\prime} j} / n\right\} \\
& =a_{i h} a_{i^{\prime} h} \sum_{k} c_{h k}^{2} \quad \text { for } \quad i, i^{\prime} \in \mathfrak{P}_{h}
\end{aligned}
$$

since $B B^{\prime}=I_{m}$. If $X$ is row-wise standardized, $\phi_{i i^{\prime}}$ and $r_{i i^{\prime}}$ are the correlation coefficients. In this context, we shall easily find out from (6) that a relationship always exists in the following ratios.

$$
\frac{\phi_{i i^{\prime}}}{\phi_{i i^{\prime \prime}}}=\frac{\phi_{i^{\prime \prime \prime} i^{\prime}}}{\phi_{i^{\prime \prime \prime} i^{\prime \prime}}}=\cdots=\frac{a_{i^{\prime} h}}{a_{i^{\prime \prime} h}}, \quad \text { for } \quad{ }^{\forall} i, i^{\prime}, i^{\prime \prime}, i^{\prime \prime \prime}, \cdots \in \mathfrak{P}_{h} .
$$

That is to say, a tetrads relationship exists for any $h$, and therefore this follows that

$$
\phi_{i i^{\prime}}=\phi_{i i^{\prime \prime}} \phi_{i^{\prime} i^{\prime \prime \prime}} / \phi_{i^{\prime \prime} i^{\prime \prime \prime}} \quad \text { for } i, i^{\prime}, i^{\prime \prime}, i^{\prime \prime \prime} \in \mathfrak{P}_{h} \text {. }
$$

This is a triad similarly called by Spearman. Thus the above formula gives us in a special case of $i=i^{\prime}$

and thereby

$$
\psi_{i i}=a_{i n}^{2} \sum_{k} c_{h k}^{2} \quad \text { for } \quad i \in \mathfrak{P}_{h}
$$

$$
\sum_{i \in \Re_{h}} \phi_{i i}=\sum_{k=1}^{m} c_{h k}^{2} .
$$

Using the above preparation, we shall now go back to estimate parameters $A, B$ and $C$ in the present model (3). Let $t_{i}$ be a sample triad defined by

$$
t_{i}=r_{i i^{\prime \prime}}, r_{i i^{\prime \prime \prime}} / r_{i^{\prime \prime} i^{\prime \prime \prime}} \quad \text { for } i, i^{\prime \prime}, i^{\prime \prime \prime} \in \mathfrak{P}_{h}, i^{\prime \prime}, i^{\prime \prime \prime} \neq i \text { (fixed), }
$$

corresponding to (8) in case of $i=i^{\prime}$. This sample triad is, of course, distributed with a triad parameter (9) and a random deviation. Such a triad parameter can be presented for all possible combinations of $i^{\prime}$ and $i^{\prime \prime}$, belonging to $\mathfrak{P}_{h}$, for a fixed $i$. The number of the combinations, say $s$, is $\left(\begin{array}{c}p_{h}-1 \\ 2\end{array}\right)$ for a triad parameter. Therefore the $s$ observed 
triads are used to obtain the least square estimate for $\psi_{i i}$. After doing this, the estimate is shown by

$$
\hat{\phi}_{i i}=\left\{\sum \sum r_{i i^{\prime \prime}} r_{i i^{\prime \prime}} / r_{i^{\prime \prime} i^{\prime \prime \prime}}\right\} / s \quad \text { for } \quad i, i^{\prime \prime}, i^{\prime \prime \prime} \in \mathfrak{B}_{h}, i^{\prime \prime}<i^{\prime \prime \prime} .
$$

By using the above estimate in (10), we can immediately obtain the estimate $\widehat{\sum_{k} c_{h k}^{2}}$ as follows,

$$
\widehat{\sum_{k} c_{h k}^{2}}=\sum_{i=\Re_{h}} \hat{\psi}_{i i}
$$

Thus the estimate of the factor loading coefficient $a_{i n}$ is given as

$$
\hat{a}_{i h}=\left(\hat{\psi}_{i i} / \widehat{\sum_{k} c_{h k}^{2}}\right)^{\frac{1}{2}} \quad \text { for } \quad{ }^{\gamma} h, i \in \mathfrak{B}_{h} .
$$

Related to the second factor loading matrix $B$, it is obvious for the relation to be dual between the parameters $a_{i n}$ and $b_{k j}$, in view of (1) and (2). Throughout the similar procedure, we obtain a sample triad as follows,

$$
\hat{\phi}_{j j}^{*}=\left\{\sum_{j^{\prime \prime}} \sum_{j^{\prime \prime}} r_{j j^{\prime \prime}}^{*} r_{j j^{\prime \prime \prime}}^{*} / r_{j^{\prime \prime} j^{\prime \prime \prime}}^{*}\right\} / s^{*} \quad \text { for } \quad j, j^{\prime \prime}, j^{\prime \prime \prime} \in \mathfrak{\Re}_{k},
$$

instead of (12), where $r_{j j^{\prime}}^{*}$ denotes a mean cross product of samples between the $j$-th and the $j^{\prime}$-th subjects and $s^{*}=\left({ }^{n_{2}-1}\right)$. Thus we obtain

$$
\hat{b}_{k j}=\left(\hat{\psi}_{j j}^{*} / \sum_{j=\Re_{k}} \hat{\psi}_{j j}^{*}\right)^{\frac{1}{2}} \quad \text { for } \quad{ }^{\forall} k, j \in \mathfrak{\Re}_{k},
$$

where the denominator is also $\widehat{\sum_{h} c_{h k}^{2}}$.

Finally we are now going to estimate the interactive factor score element $c_{h k}$ in the core matrix $C$. As it will be seen in (3), $c_{h k}$ is common in $x_{i j}$ 's, belonging to $\mathfrak{B}_{h}$ and $\mathfrak{R}_{k}$. Let $r_{i i}\left(\mathfrak{R}_{k}\right)$ be a sample cross product between the $i$-th and the $i^{\prime}$-th traits, limited for $i, i^{\prime} \in \mathfrak{P}_{h}$ and $j \in \mathfrak{N}_{k}$, and let $\phi_{i i^{\prime}}\left(\mathfrak{N}_{k}\right)$ be the corresponding parameter. This follows that

$$
\phi_{i i^{\prime}}\left(\mathfrak{N}_{k}\right)=a_{i h} a_{i^{\prime} h} c_{h k}^{2}, \quad i, i^{\prime} \in \mathfrak{P}_{h}
$$

and that

$$
\sum_{i} \phi_{i i}\left(\Re_{k}\right)=c_{h k}^{2}
$$

In case of $i=i^{\prime}$, the estimate of (17) is given by

$$
\hat{\psi}_{i i}\left(\Re_{k}\right)=\left\{\sum_{i^{\prime \prime}} \sum_{i^{\prime \prime \prime}} r_{i i^{\prime \prime}}\left(\Re_{k}\right) r_{i i^{\prime \prime \prime}}\left(\Re_{k}\right) / r_{i^{\prime \prime} i^{\prime \prime \prime}}\left(\Re_{k}\right)\right\} / s .
$$

Thus the estimate of the core element $c_{h k}$ is obtained as

$$
\hat{c}_{h k}=\left\{n_{k} \Sigma \hat{\psi}_{i i}\left(\Re_{k}\right)\right\}^{\frac{1}{2}} .
$$

It might be worth to say that the estimate (20) is equivalent, even if the procedure is starting from either side, $r_{j j^{\prime}}^{*}\left(\mathfrak{P}_{h}\right)$ or $\phi_{j j^{\prime}}^{*}\left(\mathfrak{P}_{h}\right)$, since trace $\left[\hat{\psi}_{i i^{\prime}}\left(\mathfrak{N}_{k}\right)\right]=\operatorname{trace}\left[\hat{\psi}_{j j^{\prime}}^{*}\left(\mathfrak{P}_{h}\right)\right]$. 


\section{A complete Fortran program*.}

TWO-MOOE TWO-FACTOR ANALYSIS

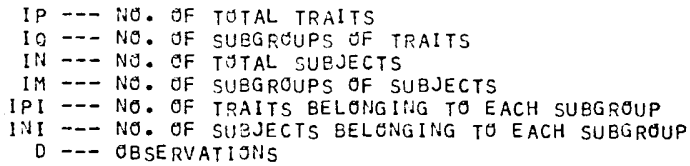

* This program was completed by using a FACOM U-200. The program language was shown here by the most basic FORTRAN, and the minimum equipments of computer system were used. 


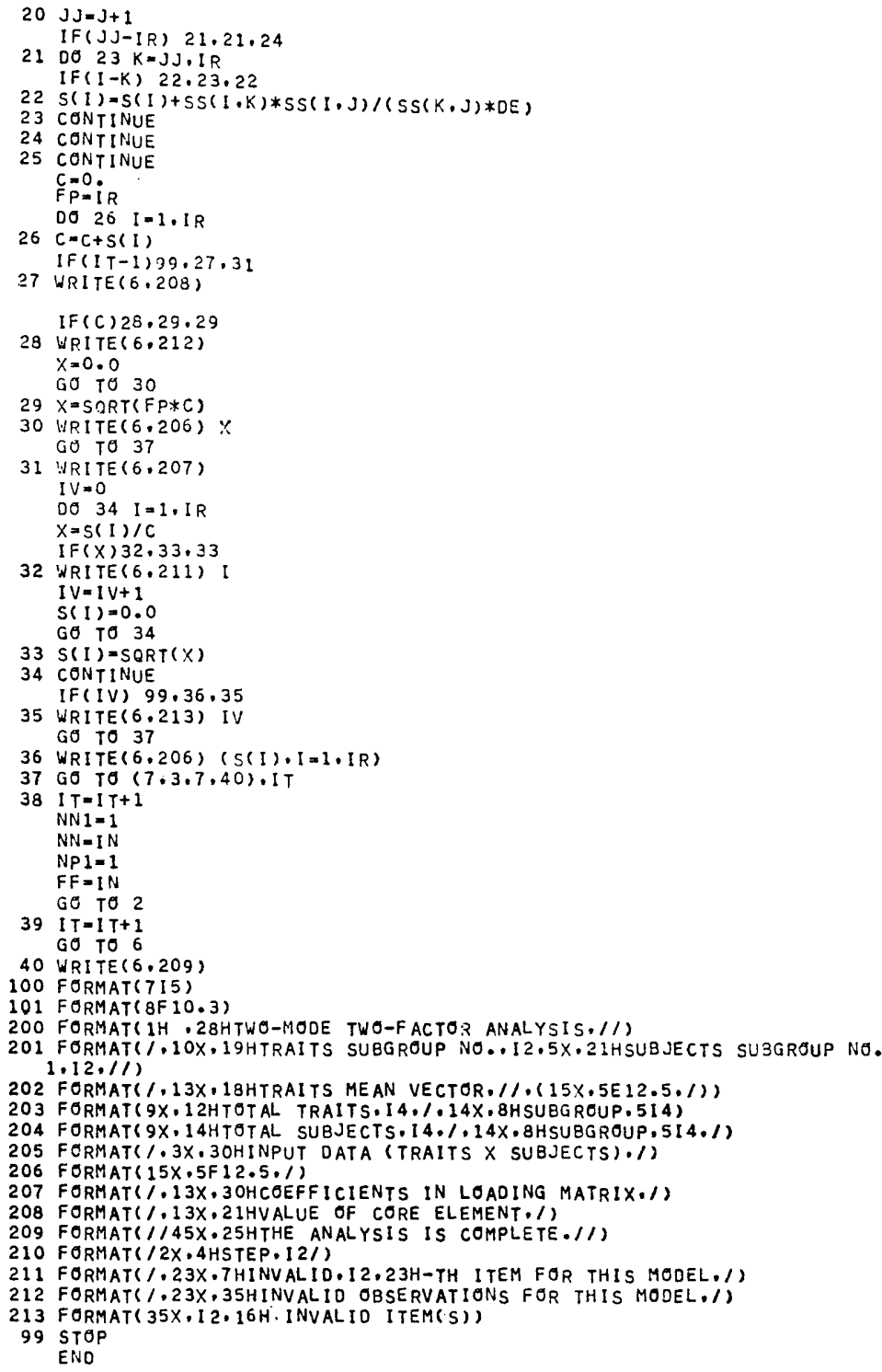

\section{Numerical illustrations.}

To illustrate numerically two-mode two-factor analysis presented here, and also to confirm the numerical result, let us consider the following example. Asano [1] studied some series of confirmation for the methods of factor analyses on a fictitious example, which was essentially reduced to rectaugles. In his data, eight traits and fifty subjects are given. Using this example, we now assume that these traits and subjects are cross-classified on the basis of physical concerns in the following way. 


\section{[Case 1]}

\section{Trait group}

$$
\begin{aligned}
& T_{1}: \mathrm{x}_{1}, \mathrm{x}_{2}, \mathrm{x}_{3}, \mathrm{x}_{4} . \\
& T_{2}: \mathrm{x}_{5}, \mathrm{x}_{6}, \mathrm{x}_{7}, \mathrm{x}_{8} .
\end{aligned}
$$

Subject group

$$
\begin{aligned}
& S_{1}: \text { (No. 's) ; } 1,3,4,5,6,7,11,12,13,14,15,16,17,18,19,20,21,24,30,44, \\
& S_{2}: \text { (No. 's) ; 8, 9, 10, 22, 23, 25, 26, 27, 28, 29, 33, 34, 45, 49, 50, } \\
& S_{3}: \text { (No. 's) ; } 2,31,32,35,36,37,38,39,40,41,42,43,46,47,48 .
\end{aligned}
$$

Then his observations $\left(x_{i j} \times 10^{-4}\right)$ are arranged in Table 1 . This means that, ahead of researcher's experiments, both sets of traits and subjects are designed and selected interactively in a cross-classification, to investigate latent factors snd their amounts involved. Actually each group of subjects $S_{i}$ is intuitively classified to three levels of the whole shapes of subjects, and is constructed independently to $T_{i}$, where $T_{i}$ is fundamentally due to the properties of test items. Thus $S_{1}, S_{2}$ and $S_{3}$ are categorized

Table 1. The design and observations for fifty subjects.

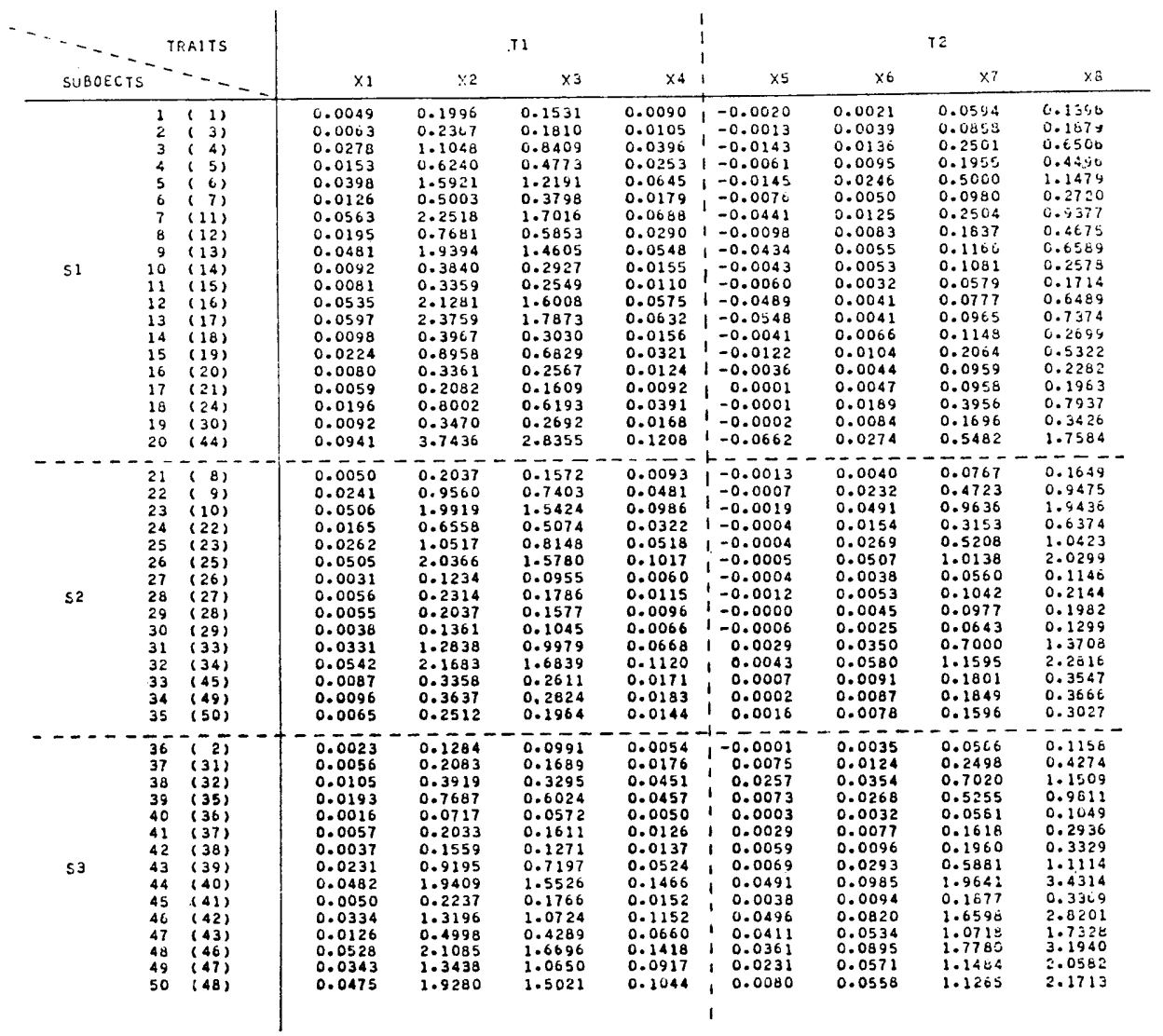


by an impression of subjects being long height, almost square and long width, respectively.

The analytical result is obtained as follows,

$$
\begin{aligned}
& \hat{A}=\left[\begin{array}{cc}
0.02 & \\
0.79 & 0 \\
0.61 & 0 \\
0.04 & \\
& 0.01 \\
\bigcap & 0.03 \\
0 & 0.57 \\
& 0.82
\end{array}\right], \quad \hat{C}=\left[\begin{array}{lll}
7.96 & 5.28 & 5.35 \\
3.34 & 4.70 & 7.76
\end{array}\right], \\
& \hat{B}^{\prime}=\left[\hat{b}_{1} \otimes \hat{b}_{2} \otimes \hat{b}_{3}\right],
\end{aligned}
$$

where $\otimes$ denotes direct product, and where

$$
\begin{aligned}
& \hat{b}_{1}^{\prime}=\left[\begin{array}{lllllllll}
0.03 & 0.04 & 0.18 & 0.11 & 0.28 & 0.08 & 0.35 & \cdots & 0.59
\end{array}\right], \\
& \hat{b}_{2}^{\prime}=\left[\begin{array}{lllllllll}
0.04 & 0.23 & 0.47 & 0.15 & 0.25 & 0.48 & 0.03 & \cdots & 0.07
\end{array}\right] \text {, } \\
& \hat{b}_{3}^{\prime}=\left[\begin{array}{lllllllll}
0.02 & 0.06 & 0.15 & 0.16 & 0.02 & 0.05 & 0.05 & \cdots & 0.36
\end{array}\right] \text {. }
\end{aligned}
$$

It will be easily able to interpret some physical meanings by the values of elements in $\hat{A}$ and $\hat{B}$. Only limiting in $\hat{C}$, we shall find out an interactive relationship between $T_{i}$ and $S_{j}$, since the values of elements in $\hat{C}$ show us a reverse trend row-wisely.

\section{[Case 2]}

We will now change only the grouping of subjcets in the same example as follows,

\section{Subject group}

$S_{1}$ : (No. 's) ; $1,2,3,5,7,8,13,14,15,20,21,26,27,28,29,31$, $36,37,38,50$,

$S_{2}$ : (No. 's) ; 4, 11, 12, 16, 17, 18, 19, 22, 24, 30, 32, 35, 41, 45, 49,

$S_{3}$ : (No. 's) ; 6, 9, 10, 23, 25, 33, 34, 39, 40, 42, 43, 44, 46, 47, 48.

This grouping is due to the global sizes of subjects, namely $S_{1}$ : slightly, $S_{2}$ : moderately, $S_{3}$ : heavily.

Then the analytical result is as follows,

$$
\begin{aligned}
& \hat{A}=[\text { all the same as in Case } 1] \text {, } \\
& \hat{C}=\left[\begin{array}{lll}
2.88 & 5.65 & 8.93 \\
1.86 & 5.95 & 8.75
\end{array}\right] \text {, } \\
& \hat{b}_{1}^{\prime}=\left[\begin{array}{llllllllll}
0.10 & 0.07 & 0.12 & 0.31 & 0.22 & 0.11 & 0.77 & 0.19 & \cdots & 0.16
\end{array}\right] \text {, } \\
& \hat{b}_{2}^{\prime}=\left[\begin{array}{llllllllll}
0.26 & 0.48 & 0.18 & 0.42 & 0.47 & 0.10 & 0.21 & 0.18 & \cdots & 0.10
\end{array}\right] \text {, } \\
& \hat{b}_{3}^{\prime}=\left[\begin{array}{llllllllll}
0.18 & 0.13 & 0.27 & 0.14 & 0.28 & 0.18 & 0.30 & 0.14 & \cdots & 0.28
\end{array}\right] \text {. }
\end{aligned}
$$


In view of $\hat{C}$, it seems to us that the row-wise values in $C$ are comparatively parallel. This suggests us that $T_{1}$ and $T_{2}$ do not show any interaction to $S_{1}, S_{2}$ and $S_{3}$, excepting the respective factors in subject groups and trait groups.

Finally it will be convenient to confirm the results for us to see the true patterns of subjects in this illustration by rectangles like in Figure 1.

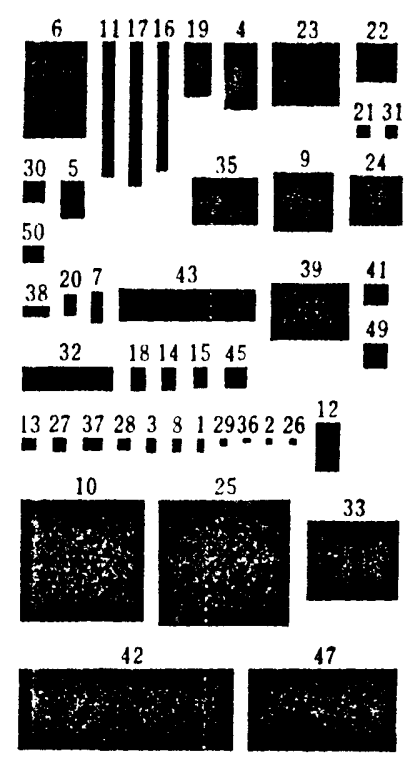

46
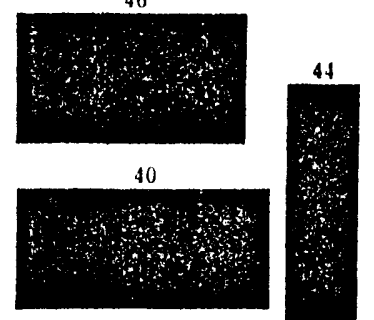

34

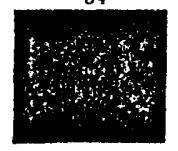

48

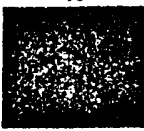

Figure 1. The true patterns only God knows for subjects.

\section{References}

[1] C. Asano, Factor analyses, (in Japanese), Kyoritsu-shuppan (1971), 30-32.

[2] J. Levin, Three-mode factor analysis, Tech. Rept., Dept., Psychol. Univ. Illi. (1963).

[3] J. Levin, Three-mode factor analysis, Psychol. Bull. 64 (1965), 442-452.

[4] C.E. Osgood, G. J. Suci and P.H. TAnnenbaum, The measurement of meaning, Urbana, Illinois, Univ. Ill. Press (1957).

[5] L.R. TUCKER, Implication of factor analysis of three-way matrices of measurement of change, in C.W. Harris (Ed): Problems in measuring change, Madison, Univ. Wiss. 
Press (1963), 122-137.

[6] L.R. TUCKER, The extension of factor analysis to three dimensional matrices, in $\mathrm{N}$. Frederikson and H. Gulliksen (Ed): Contributions to mathematical psychology, New York Holt, Rinehart and Winston (1964), 109-127.

[7] L.R. Tucker, Experiments in multi-mode factor analysis, Proceeding of the 1964 Invi. tational Conference on Testing Problems, N. J. Educ. Test Serv. (1965), 46-57.

[8] L.R. Tucker, Some mathematical notes on three-mode factor analysis, Psychometrika, 31 (1966), 3, 279-311. 